\title{
Monitoring gears by vibration measurements: Lempel-Ziv complexity and Approximate Entropy as diagnostic tools
}

\author{
Mourad Kedadouche, Marc Thomas ${ }^{\mathrm{a}}$, Antoine Tahan and Raynald Guilbault \\ Department of Mechanical Engineering, École de Technologie Supérieure 1100, Notre-Dame street West, Montreal, \\ H3C 1K3, Quebec, Canada
}

\begin{abstract}
Unexpected failures of industrial gearboxes may cause significant economic losses. It is therefore important to detect early fault symptoms. This paper introduces signal processing methods based on approximate entropy (ApEn) and LempelZiv Complexity $(L Z C)$ for defect detection of gears. Both methods are statistical measurements exploring the regularity of a vibratory signal. Applied to gear signals, the parameter selection of $A p E n$ and $L Z C$ calculation are first numerically investigated, and appropriate parameters are suggested. Finally, an experimental study is presented to investigate the effectiveness of these indicators. The results demonstrate that $A p E n$ and $L Z C$ provide alternative features for signal processing. A new methodology is presented combining both Kurtosis and $L Z C$ for early detection of faults. The results show that this proposed method may be used as an effective tool for early detection of gear faults.
\end{abstract}

\section{Introduction}

Gearboxes play an important role in industrial applications, and unexpected failures often result in significant economic losses. Numerous papers considering gear condition monitoring through vibration measurements were published over the years. Compared to classical techniques such as statistical time indicators or Fast Fourier Transform, advanced signal processing techniques like time-frequency analysis (STFT, Wigner-Ville) [1-4] or wavelet transform $[5,6]$ have shown to be more efficient for gear defect detection. Baydar et al. [7-10] proposed various methods such as the instantaneous power spectrum, Wigner-Ville distribution and the wavelet transform method for local tooth fault detection from vibration and acoustic signals. Yesilyurt [11] applied the spectrogram and scalogram approach for gearbox fault detection.

The Lempel-Ziv complexity (LZC) and approximate entropy $(A p E n)$ methods present alternative tools for signal analysis involving nonlinear dynamics. These methods are becoming popular and have found wide applications in various disciplines, especially in the field of biomedical engineering. The ApEn method has recently received more attention. Yan [12] investigated the application of ApEnfor the health monitoring of rolling element bearings. Y. He and X. Zhang [13] applied the ApEn method for monitoring acoustic emission signals from defects in rolling element Bearings. Fu et al. [14] used the ApEn method to fault signal analysis in electric power system. UsingApEn, Xu et al. [15] detected the looseness of the bearing bushing in turbo generator. In all these works, $A p E n$ was used as a nonlinear feature parameter to analyse the vibration signal for effectively identifying the conditions of the mechanical system.

\footnotetext{
${ }^{a}$ Corresponding author: marc . thomas@etsmtl.ca
}

On the other hand, Yan and Gao [16] investigated the application of Lemp-Ziv complexity $(L Z C)$ for the health monitoring of rolling element bearings. Wang et al. [17] compare and analyse quantitative diagnosis methods based on Lempel-Ziv complexity for bearing faults, using continuous wavelet transform (CWT), Empirical Mode Decomposition (EMD) method, and wavelet packet method for decomposition of vibration signal. Kedadouche et al. [18] combined LZC and EMD for early detection of gears cracks.

As illustrated above, the $L Z C$ and ApEn methods are becoming more and more attractive in the field of detection and fault diagnosis. However, no work has been found to apply ApEn or $L Z C$ measurement for gear faults diagnosis. Therefore, in this paper, the ApEn and $L Z C$ methods are compared in order to analyse vibration signals from gear and investigate their efficiency for the defect detection and severity evaluation of gears faults.

\section{Theoretical background}

\subsection{Approximate entropy}

Consider a time series $S(i), i=0 \ldots N$. Its "regularity" may be measured by ApEn in a multiple dimensional space so that series of vectors are constructed and expressed as follows:

$$
\begin{aligned}
X(N-m+1)=\{ & x(N-m+1), \\
& x(N-m+2), \ldots, x(N)\} .
\end{aligned}
$$

Each vector is composed of $\mathrm{m}$ consecutive and discrete point data of the time series $S$. The distance between two vectors $X(i)$ and $X(j)$ can be defined as the maximum

This is an Open Access article distributed under the terms of the Creative Commons Attribution License 2.0, which permits unrestricted use, distribution, and reproduction in any medium, provided the original work is properly cited. 
difference in their respective corresponding elements:

$$
\begin{array}{r}
d(X(i), X(j))=\max _{k=1,2, . . m}(\mid x(i+k-1) \\
-x(j+k-1) \mid)
\end{array}
$$

where $i=1,2 \ldots, N-m+1, j=1,2 \ldots, N-m+1$, $N$ is the number of data points contained in the times series.

For each vectors $X(i)$, a measurement that describes the similarity between the vectors $X(i)$ and all other vectors $X(j)$ can be defined as:

$$
C_{i}^{m}(r)=\frac{1}{N-(m-1)} \sum_{j \neq i} \Theta\{r-d(X(i), X(j))\}
$$

where

$$
\Theta\{x\}=\left\{\begin{array}{l}
1, x \geq 0 \\
0, x<0
\end{array}\right.
$$

The symbol $r$ represents a predetermined tolerance value, where $k$ is a positive constant $(k>0)$ and $s t d_{S}$ is the standard deviation of the time series $S$.

$$
r=k \times s t d_{S}
$$

Finite time series consisting of $N$ data points are used to estimate the ApEn value of the time series, which is defined as:

$$
\begin{gathered}
\operatorname{ApEn}(m, r, N)=\emptyset^{m}(r)-\emptyset^{m+1}(r) \\
\emptyset^{m}(r)=\frac{1}{N-m+1} \sum_{i=1}^{N-m+1} \ln \left[C_{i}^{m}(r)\right] .
\end{gathered}
$$

\subsection{Complexity analysis}

The complexity analysis is based on the Lempel-Ziv definition [19]. This approach transforms the analysed signal into a data sequence. To illustrate the procedure, consider a gear vibration signal with a known mean value. A new sequence $(S)$ is reconstructed by comparing the value of each sample of the previous sequence within the mean value. If the value of the sample is larger, it is set to one (1), otherwise to zero (0). Therefore, only two binary symbols are present in the new data sequence. This $\mathrm{S}$ is subsequently scanned from its first sample to its end. When a subsequence that is not encountered in the previous scanning process is discovered, the complexity value is increased by one (1). Thus, the Lempel-Ziv complexity reflects the number of all different subsequences contained in the original sequence. Figure 1 (reproduced from [16]) described the algorithm. For generality sake, normalized complexity $C(n)$ is often used to obtain a measure independent of the sequence length.

$$
\begin{gathered}
C(n)=c(n) / b(n) \\
b(n)=N / \log 2(N) .
\end{gathered}
$$

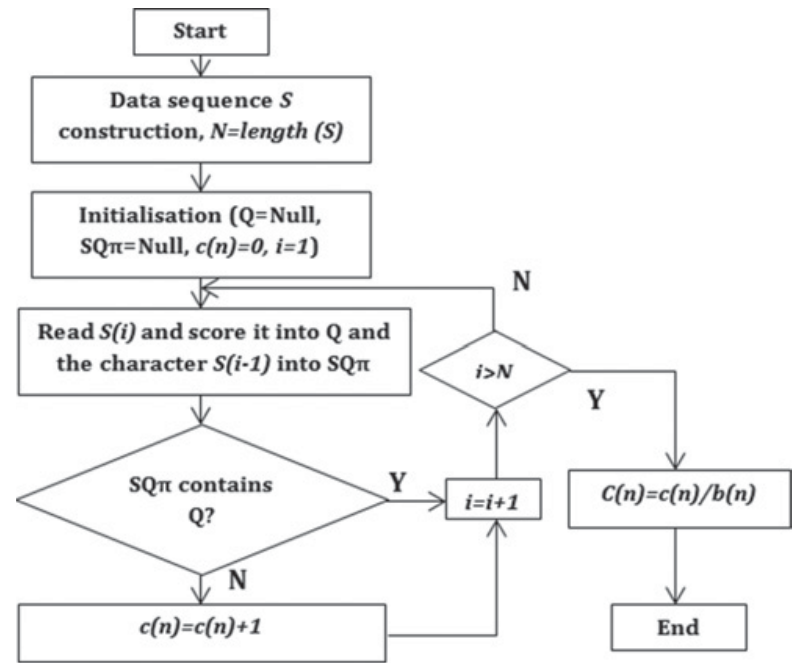

Figure 1. The flow chart of $L Z C$ algorithm.

Table 1. Geared system data of the simulated signal.

\begin{tabular}{|l|l|l|}
\hline & $\begin{array}{l}\text { Frequency } \\
(\mathrm{Hz})\end{array}$ & $\begin{array}{l}\text { Ampli- } \\
\text { tude }\end{array}$ \\
\hline Pinion: Number of teeth $=20$ & 15 & 0.15 \\
\hline Gear: Number of teeth $=21$ & 14.28 & 0.15 \\
\hline Gear mesh: The First Harmonic & 300 & 1 \\
\hline Gear mesh: The Second Harmonic & 600 & 0.6 \\
\hline Gear mesh: The Third Harmonic & 900 & 0.3 \\
\hline
\end{tabular}

\subsection{Parameters Selection of ApEn and $L Z C$ for gear Signals}

From above described algorithm of $A p E n$ method, it can be seen that the calculated $A p E n$ value depends on two parameters, which are the embedded dimension $m$ and the tolerance r. However, no guideline exists for optimizing theses values. In order to simulate the vibratory signals of gearbox, a gear multiplicative model whose the meshing is modulated in amplitude has been used. The gear model as defined in [20] is used (Table 1):

$$
\begin{aligned}
x(t) & =\sum_{m=-\infty}^{+\infty} S_{r 1}\left(t-m \tau_{r 1}\right)+\sum_{m=-\infty}^{+\infty} S_{r 2}\left(t-m \tau_{r 2}\right) \\
& +\sum_{n=-\infty}^{+\infty} S_{e}\left(t-n \tau_{e}\right) \cdot\left(1+\sum_{m=-\infty}^{+\infty} S_{r 1}\left(t-m \tau_{r 1}\right)\right)
\end{aligned}
$$

where $\tau_{e}, \tau_{r 1}$ and $\tau_{r 2}$ represent the meshing period and the rotational periods. $S_{e}(t), S_{r 1}(t)$ and $S_{r 2}(t)$ represents the meshing signal and its modulation.

Figure 2 and Fig. 3 represent the simulated signal and its spectrum, respectively.

For a given dimension $m$, the key step of the ApEn algorithm is to calculate the distance between two vectors and to count the number of distance less than $r$ (where $\mathrm{r}=\mathrm{k}^{*}$ standard deviation of the time series), as discussed in [12-16]. The selections of $\mathrm{k}$ and $\mathrm{m}$ are based on a convergence analysis. The choice of $\mathrm{m}$ and $\mathrm{k}$ depends on the nature of the time series treated. The best parameters 


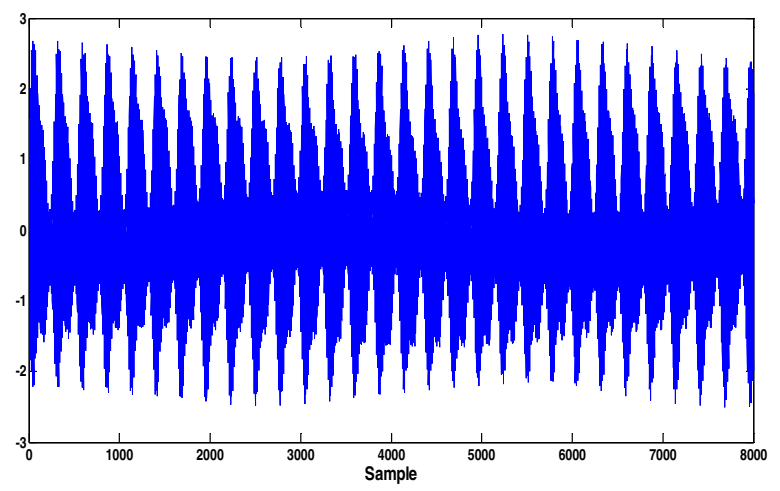

Figure 2. The simulated signal.

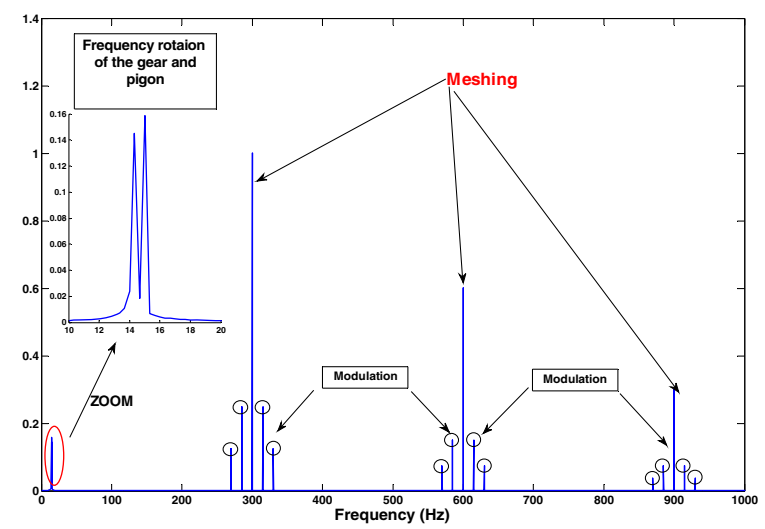

Figure 3. Spectrum of the simulated signal.

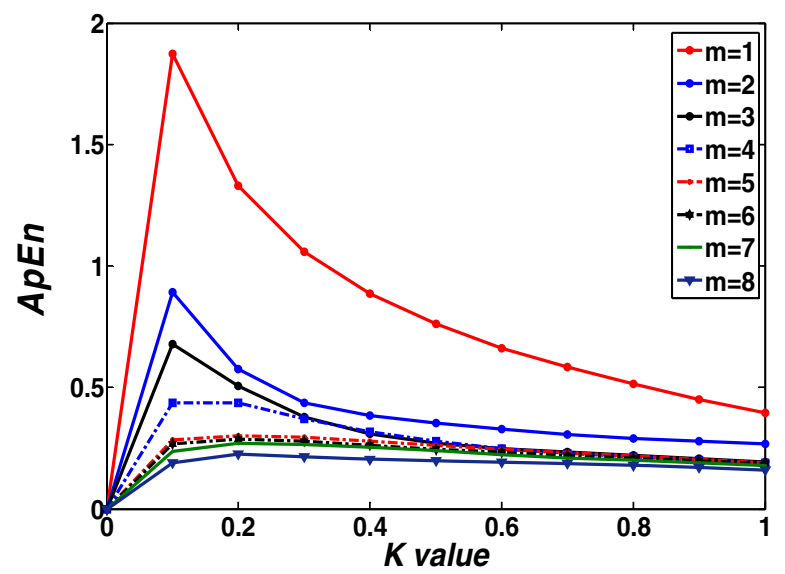

Figure 4. The calculated ApEn values by different parameters. (Kvalue and $m$ ).

to be chosen are those who present little fluctuation, and thus a good reproducibility. From Fig. 4, it can be seen that, when $\mathrm{m}=1$, ApEn exhibits the poorest convergence property (a large fluctuation). With $\mathrm{m}$ increasing (more than 2), the convergence property of ApEn becomes better for $\mathrm{k}$ greater than 0.4. However, a larger $\mathrm{m}$ will lead to much higher computational cost. In this study, a convergence property of ApEn is assumed already good enough with $\mathrm{m}=2$. Of course, the $\mathrm{k}$ value should also be selected as a compromise to avoid losing much detailed system information.

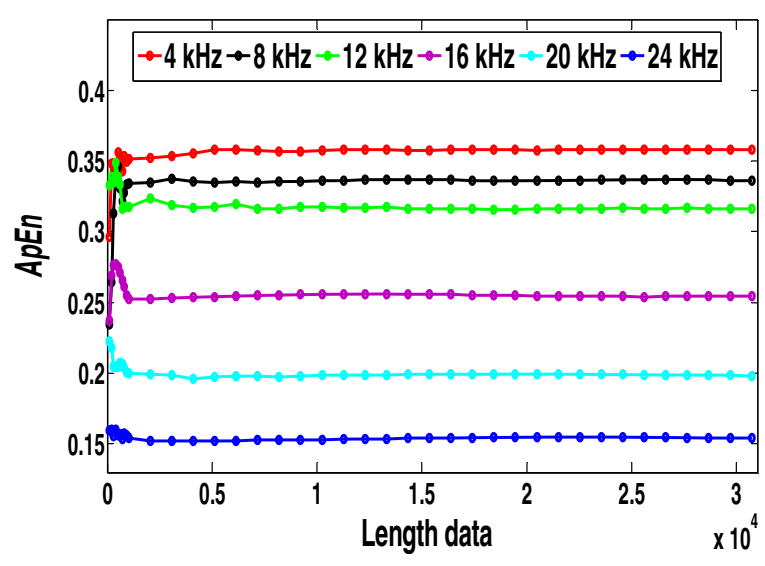

Figure 5. The calculated ApEn values by different parameters. (Length of the data and the sample frequency).

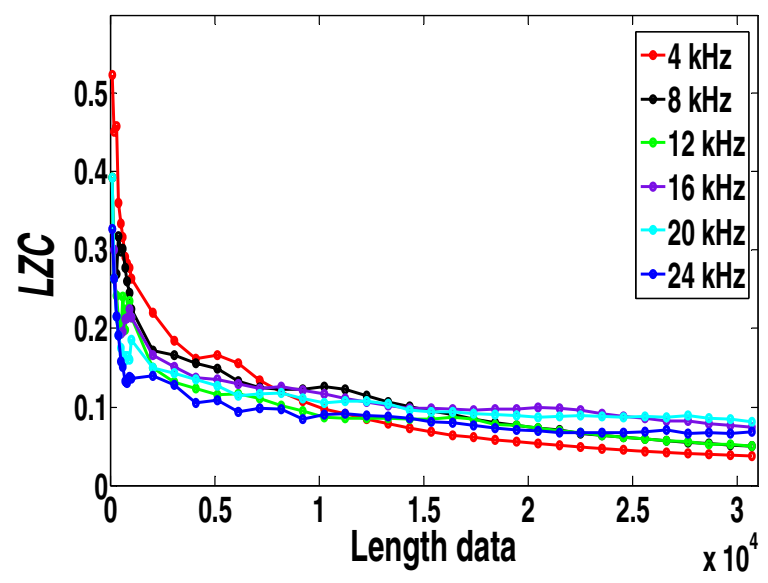

Figure 6. The calculated $L Z C$ values by different parameters. (Length of the data and the sample frequency).

From this investigation, $m=2$ and Kvalue $=0.5$ times the standard deviation are selected for the ApEn calculation of the vibration signal.

The relationship between the ApEn, LZC value and the data length is illustrated in Fig. 5 and Fig. 6, where seven simulated signals are comparatively displayed, under sampling rates of 4, 8, 12, 16, 20 and $24 \mathrm{kHz}$, respectively.

It is seen, in both cases (ApEn and $L Z C)$, that when the data length is greater than 5000 points, the variation of ApEn and $L Z C$ with respect to each frequency sampling rate become insignificant. However, the sampling frequency and the data length are linked. So we must take consideration of the components defining the signal. To respect the Shannon theorem, the sample frequency must be more than 2 times the highest frequency presented in the signal. In our case, the sample frequency must be greater than 2 time the third meshing frequency $(900 \mathrm{~Hz})$.

The simulation where conducted with $F_{s}$ greater than $4 \mathrm{kHz}$ which is sufficient. For a better detection of the modulation, we must chose a good resolution frequency which is defined by $\Delta H=F_{s} /$ length(signal). In the simulated signal, the modulations are about $\pm 15 \mathrm{~Hz}$. So, a frequency resolution of $1 \mathrm{~Hz}$ is enough. This means that the minimum length of data to be analysed is equal to the sample frequency $(4000 \mathrm{~Hz})$. 


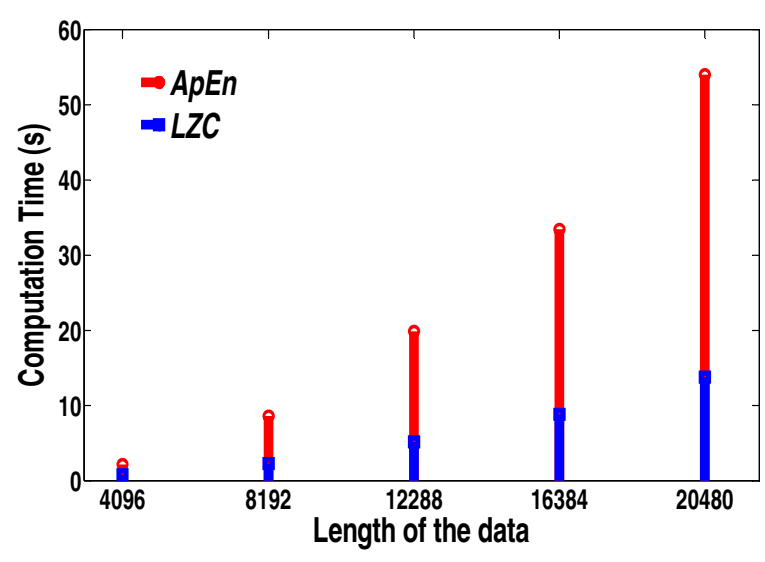

Figure 7. Time Computation of both $L Z C$ and ApEnfor different length of data.

Table 2. ApEn and $L Z C$ for different SNR of gear signals.

\begin{tabular}{|l|l|l|}
\hline Cases & ApEn & LZC \\
\hline simulated signal (no noise) & 0.3529 & 0.1294 \\
\hline $\mathrm{SNR}=80 \mathrm{~dB}$ & 0.3547 & 0.1364 \\
\hline $\mathrm{SNR}=60 \mathrm{~dB}$ & 0.3648 & 0.1692 \\
\hline $\mathrm{SNR}=40 \mathrm{~dB}$ & 0.4495 & 0.3056 \\
\hline $\mathrm{SNR}=0 \mathrm{~dB}$ & 1.3163 & 0.8909 \\
\hline
\end{tabular}

Figure 7 shows a comparison of computing time between $L Z C$ and ApEn. It is clear that the $L Z C$ is better than ApEn.

\subsection{Influence of noise}

It is well known that a white noise excites all the frequency components compared with other kinds of signal. If a signal is contaminated by a white noise, calculated ApEn and $L Z C$ values will also be. Using the simulated signal, the ApEn and $L Z C$ values corresponding to different SNRs were calculated, as listed in Table 2.

It may be noticed that the ApEn and $L Z C$ values increase as the SNR decreases, which corresponds to a degradation of the data quality. As discussed in [12], the working conditions of machine system deteriorate due to the degradation of the defects. The number of frequency components contained in the signal will increase and the SNR decrease, resulting in a decrease in its regularity. This allows a decrease of its $A p E n$ and $L Z C$ values. The simulation results listed in Table 2 confirms that the ApEn and $L Z C$ values provide a quantitative measurement for characterising a dynamic signal which can be represented by the deterioration of a machine's health condition. From Table 2, it can also be seen that ApEn is very sensitive to large noises; however, $A p E n$ is nearly unaffected by the noise which it stays low. Otherwise, we can see that the $L Z C$ is more sensitive to noise than ApEn.

\section{Experimental study}

The recordings of vibration signals were carried out at CETIM, France on a gear system with a train of gearing, with a ratio of 20/21 functioning continuously until its destruction. The sample frequency was set to $20 \mathrm{kHz}$.
Table 3. Geared system data.

\begin{tabular}{|l|l|l|}
\hline Parameter & Pinion & Gear \\
\hline Number of tooth & 20 & 21 \\
\hline Speed $(\mathrm{rpm})$ & 1000 & 952.38 \\
\hline Drive torque $(\mathrm{Nm})$ & 200 & \\
\hline Face widh $(\mathrm{m})$ & 0.015 & 0.03 \\
\hline Module $(\mathrm{m})$ & 0.01 & 0.01 \\
\hline Pressure angle & $20^{\circ}$ & $20^{\circ}$ \\
\hline Addendum coefficient & 1.0 & 1.0 \\
\hline Dedendum coefficient & 1.4 & 1.4 \\
\hline
\end{tabular}

Table 4. Daily mechanical appraisal.

\begin{tabular}{|l|l|}
\hline Day & Observation \\
\hline 1 & No acquisition \\
\hline 2 & No anomaly \\
\hline 3 & No anomaly \\
\hline 4 & // // \\
\hline 5 & // / \\
\hline 6 & // // \\
\hline 7 & Chipping teeth $1 / 2$ \\
\hline 8 & No evolution \\
\hline 9 & Tooth $1 / 2:$ no evolution; Tooth $15 / 16:$ start chipping \\
\hline 10 & Evolution of the chipping of the teeth $15 / 16$ \\
\hline 11 & // // \\
\hline 12 & // // \\
\hline 13 & Chipping across the full width of the tooth \\
\hline
\end{tabular}

Table 3 gives the details of the gear test rig parameters. The test duration was 13 days with a daily mechanical appraisal; measurements were collected every $24 \mathrm{~h}$ except at the first day. Table 4 gives a description of the state of the gear at each $24 \mathrm{~h}$. The acceleration signals for days 2 , 5, 7, 9, 10 and 12 are shown in Fig. 8.

Figure 9 presents some pictures of the chipping observed in day 11 and 12 .

Pareya et al. [21] use the same signal for their own research. Only the Kurtosis and Crest Factor were considered. The Kurtosis values for the experimental signal were calculated from day 2 to 13 and are shown in Fig. 10 and Fig. 11 (on day 1 no signal was taken).

It can be seen that the Kurtosis increases greatly after the day 11. This indicates that the signal becomes impulsive. The Crest Factor observes a little increase after the day 9 (2.98 to 3.60). This is due to the evolution of the chipping of the teeth $15 / 16$ observed in day 9 .

The values of ApEn and LZC for all signal are plotted in Fig. 12 and Fig. 13. The method ApEn doesn't present a significant increase except at the Day 12. So the characteristic ApEn seems to have equivalent efficiency as Kurtosis or Crest Factor.

On the other hand, LZC shows a significant increase after the day 4, revealing a clear anomaly due to gear wear. However, it is not clear why a decrease at days 10 and 11 is observed. We may suspect that the signal was noisier at these measurements.

Figure 14 presents both the kurtosis and $L Z C$ of the day 12. The Kurtosis is reported in the abscise $X$ and $L Z C$ on $\mathrm{Y}$. 

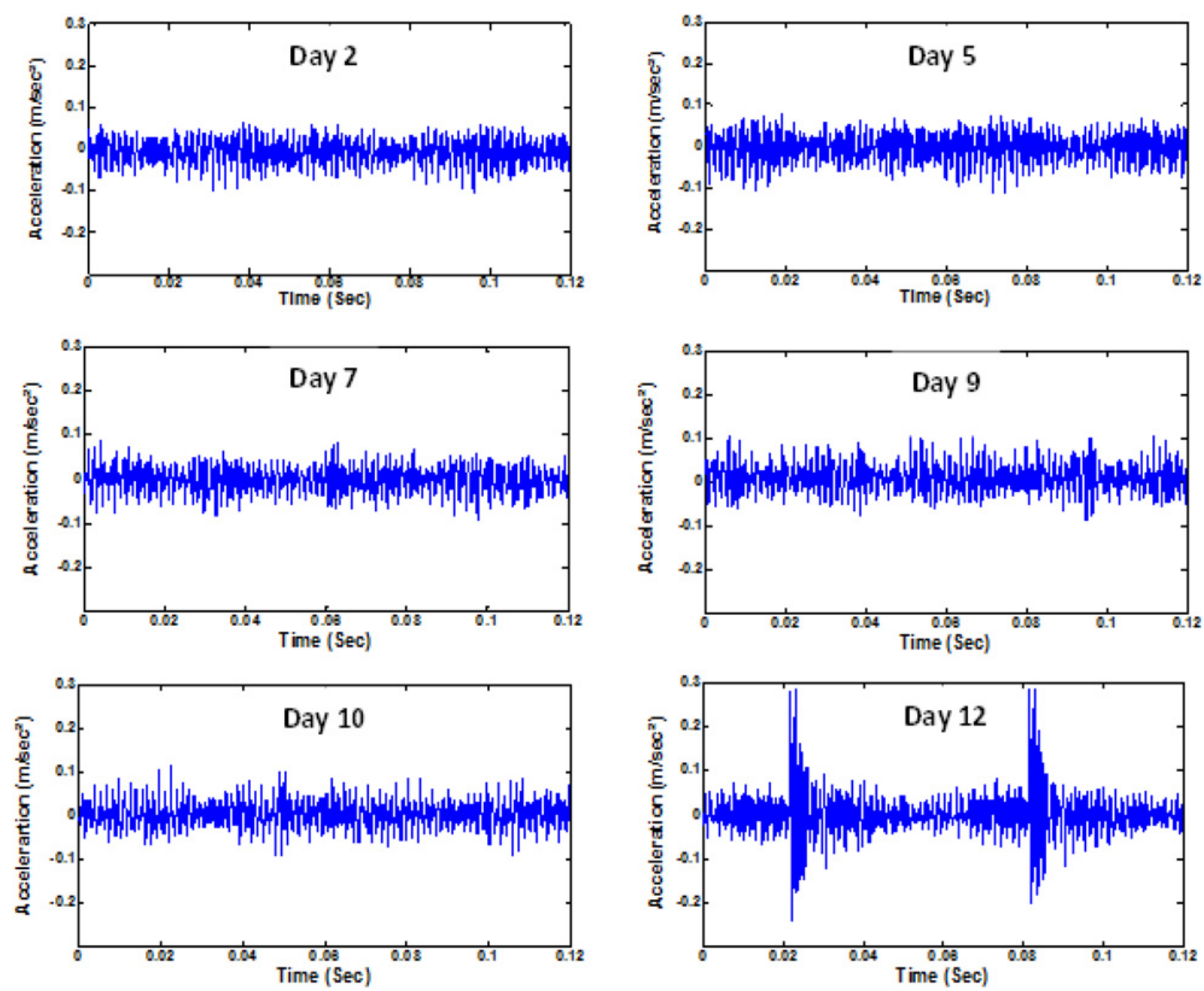

Figure 8. Evolution of acceleration signal with wear.

Dent 2 dav 11

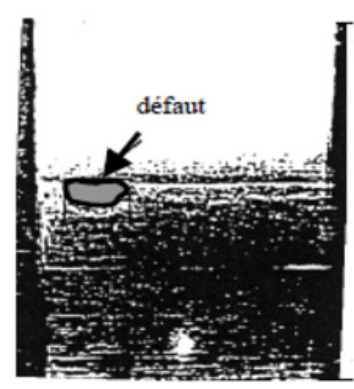

Dent 16, day 11

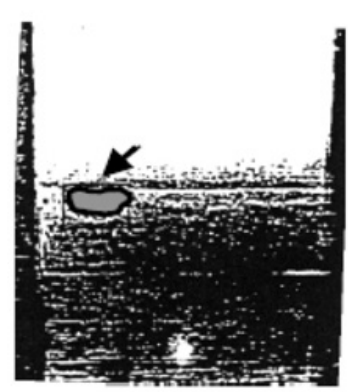

Dent 16 day 12

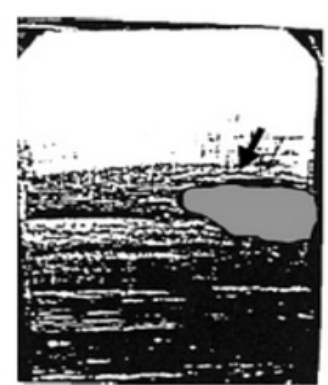

Figure 9. Evolution of the chipping (from CETIM).

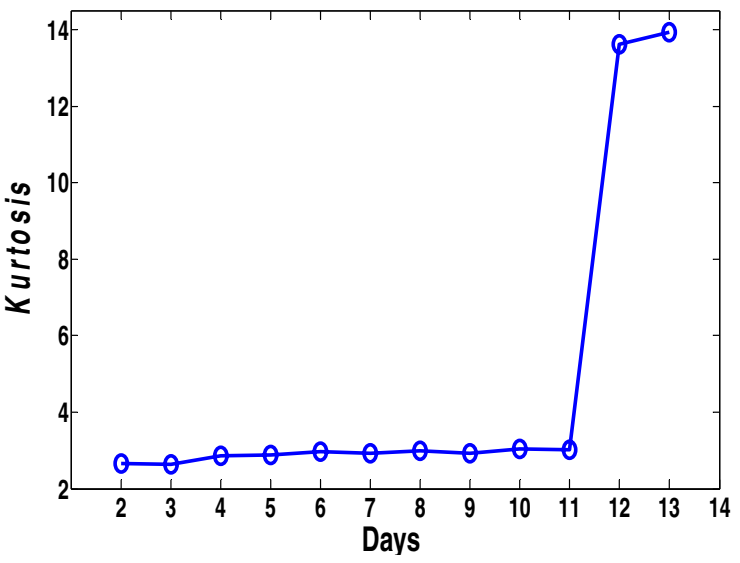

Figure 10. Kurtosis Value during the test for all days [from 21].

This representation gives both information on the impulsiveness of the signal and on effect of the number of frequency components and noise into the signal. It can be seen that this plot divides the twelve days into three regions. The first region contains the three first days.

The second enclose all days from 6 to 11. The second region is separated from the first by the day 5 . The day 5 present the change in the characteristics of the gear signal. 


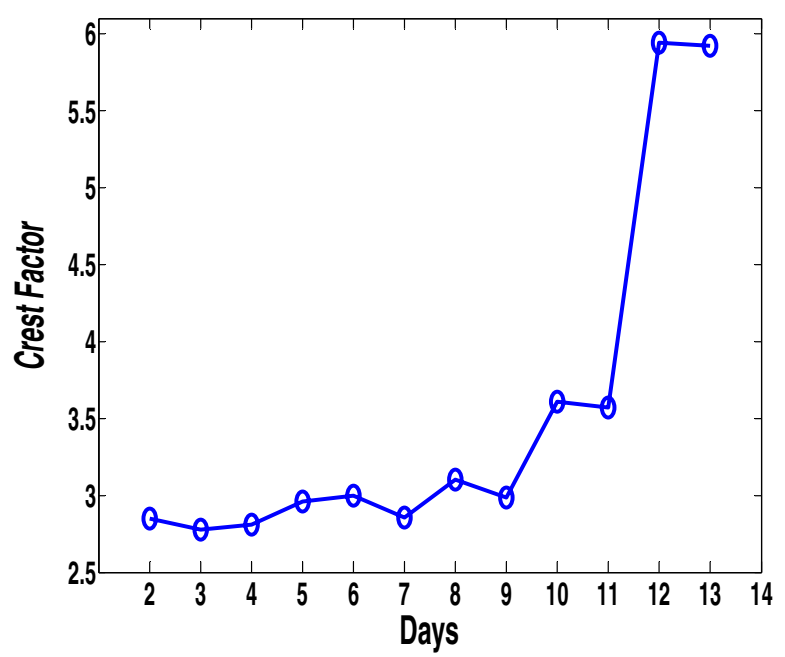

Figure 11. Crest Factor Value during the test for all days [from 21].

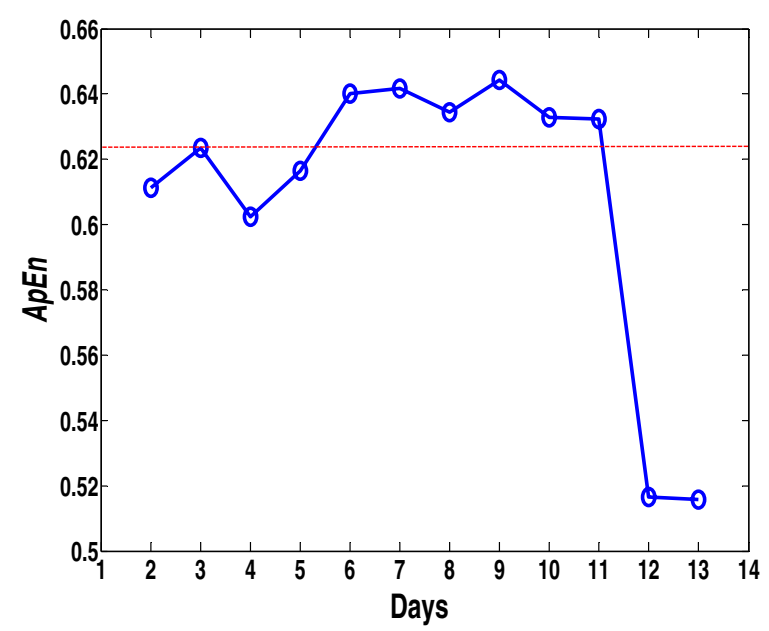

Figure 12. Crest Factor Value during the test for all days.

Figure 15 compares the spectrum evolution between days 4 and 5 . An increase of the amplitude at day 5 may be clearly noticed as compared with the day 4. Effectively, this day presents the beginning of the degradation due to wear. The last region is marked by an increase of the Kurtosis and the stays in the same level as the second region. At this stage, the signal becomes impulsive and the gear is damaged.

According to Table 4, the chipping has only visually been observed after the day 6. According to $L Z C$ measurements, the beginning of the chipping was in fact initiated at the day 5. This initiation is characterised by the growth of the frequency components related to the meshing. Consequently, this representation combining Kurtosis and $L Z C$ may be used as an efficient tool for early detection of faults.

\section{Conclusion}

This paper introduces ApEn and $L Z C$ metrics to analyse the vibration signals recorded from defected gears. With respect to gear signals, the parameter selection of $A p E n$ was investigated and the results show that $m=2$ and

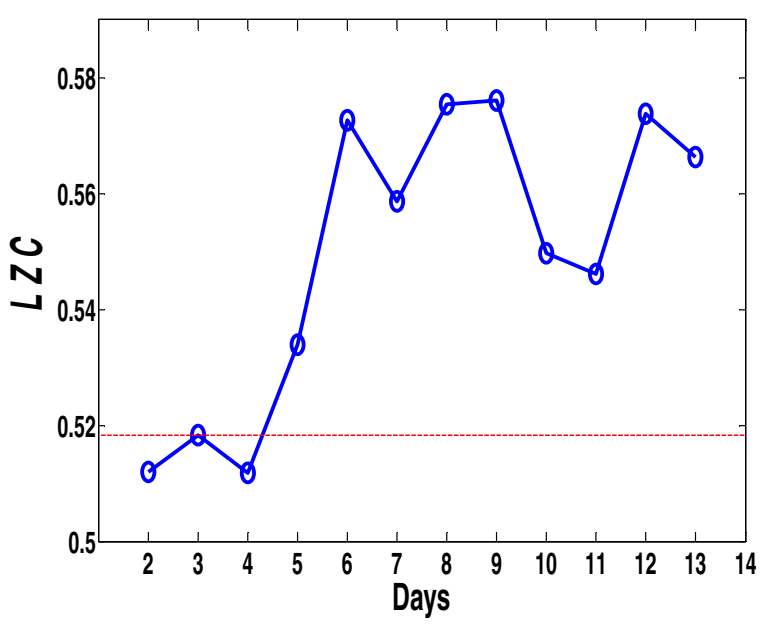

Figure 13. LZC Values during the test for all days.

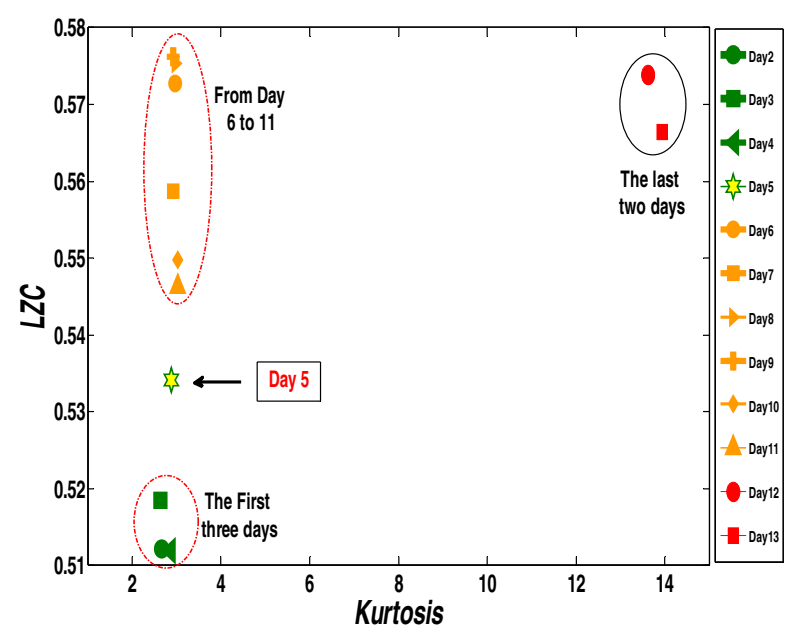

Figure 14. Kurtosis versus LZC Value during the test for all days.

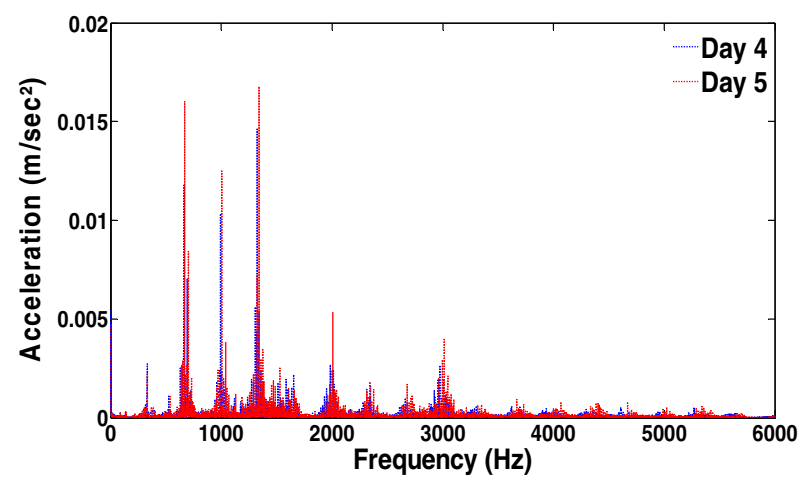

Figure 15. Spectrum of the experimental signal.

Kvalue $=0.5$ times the standard deviation were suitable and a good compromise for the ApEn calculation in the investigated application. In addition, the influence of white noise on the $A p E n$ and $L Z C$ calculation was also investigated. The results show that $A p E n$ is nearly unaffected by the noise when staying at a small level. However, ApEn is very sensitive to the noise at high levels. The $L Z C$ is more sensitive to noise as compared with ApEn. An experimental study was conducted to evaluate the effectiveness of these parameters. The results show 
that the LZC and ApEn can detect the defect of the gears earlier than the classical temporal indicators. However These preliminary studies suggest that the $L Z C$ appeared more efficient than $A p E n$. The representation in a plan (Kurtosis, $L Z C$ ) is proposed as a new tool for effectively monitoring gear defects. Although experimental results look promising, the proposed vibration methodology has yet to be tested on other test rigs. The research is thus being continued to analyze vibration signals from different defect types and on different types of gears, to systematically validate the efficiency of this technique.

The financial support of NSERC (Natural Sciences and Engineering Research Council of Canada), FQRNT (Fonds Québécois de la Recherche sur la Nature et les Technologies), MITACS Canada, Pratt \& Whitney Canada and CETIM which provided the experimental results are gratefully acknowledged.

\section{References}

[1] Wang WJ and McFadden PD. Early detection of gear failure by vibration analysis $-\mathrm{I}$. Calculation of the time-frequency distribution. MSSP, 7(3): 193-203 (1993)

[2] Wang WJ and McFadden PD. Early detection of gear failure by vibration analysis -II. Interpretation of the time-frequency distribution using image processing techniques. MSSP; 7(3): 205-15 (1993)

[3] Safizadeh M.S., Lakis A.A. and Thomas M., Gear Fault Diagnosis using time-frequency methods, proceedings of 20th seminar on machinery vibration, Canadian Machinery Vibration Association, Quebec, 7.19-7.29 (2002)

[4] Safizadeh M.S., Lakis A.A. and Thomas M., Using Short-Time Fourier Transform in Machinery diagnosis, Proceedings of WSEAS (Brazil) 494-200, (2005)

[5] Z.K. Peng, F.L. Chu, Application of the wavelet transform in machine condition monitoring and fault diagnostics: a review with bibliography, Mechanical Systems and Signal Processing 18, 199-221 (2004)

[6] R. Yan, R. X. Gao and X. Chen, Wavelets for fault diagnosis of rotary machines: A review with applications, Signal Processing 961-15 (2014)

[7] Baydar N and Ball A. Detection of gear deterioration under varying load conditions by using the instantaneous power spectrum. Mechanical Systems and Signal Processing; 14(6): 907-21 (2000)

[8] Baydar N, Chen Q, Ball A, Kruger U. Detection of incipient tooth defect in helical gears using multivariate statistics. MSSP 15(2): 303-21 (2001)

[9] Baydar N., Ball A. A comparative study of acoustic and vibration signals in detection of gear failures using Wigner-Ville distribution. Mechanical Systems and Signal Processing 15(6): 1091-1107 (2001)

[10] Baydar N. and Ball A. Detection of gear failures via vibration and acoustic signal using wavelet transform. Mechanical Systems and Signal Processing, 17(4): 787-804 (2003)

[11] Yesilyurt I., The application of the conditional moments analysis to gearbox fault detectiona comparative study using the spectrogram and scalogram, NDT\&E International 37, 309-320 (2004)

[12] Yan, R., and Gao, R. X., "Approximate Entropy as a Diagnostic Tool for Machine Health Monitoring," Mechanical Systems and Signal Processing 21, 824-839 (2007)

[13] He Y. and X. Zhang. Approximate Entropy Analysis of the Acoustic Emission From Defects in Rolling Element Bearings, Journal of Vibration and Acoustics, 134 / 061012-1 (2012)

[14] Fu, L., He, Z. Y., Mai, R. K., and Qian, Q. Q., Application of Approximate Entropy to Fault Signal Analysis in Electric Power System, Proceedings of the Chinese Society of Electric Engineering, 28(28), 68-73 (2008)

[15] Xu, Y. G., Li, L. J., and He, Z. J. Approximate Entropy and its Applications in Mechanical Fault Diagnosis, Chin. J. Inf. Control, 31(6), 547-551 (2002)

[16] R. Yan and R. X. Gao, Complexity as a Measure for Machine Health Evaluation, IEEE transactions on instrumentation and measurement, 53(4), 1327-1334 (2004)

[17] Wang J., L. Cui, H. Wang and P. Chen , Improved Complexity Based on Time-Frequency Analysis in Bearing Quantitative Diagnosis, Advances in Mechanical Engineering, Article ID 258506, 11 pages http://dx.doi.org/10.1155/2013/258506 (2013)

[18] Kedadouche M., Kidar T., Thomas M. and Tahan A. , Combining EMD and Lempel-Ziv Complexity for early detection of gear cracks, Surveillance 7, Chartres, France, pp 100-110, 28-29 (2013)

[19] Lempel A. and Ziv J., On the complexity of finite sequences, IEEE Trans. Inform. Theory,Jan. IT22, 75-81 (1976)

[20] El Badaoui, M.: Contribution of vibratory diagnostic of gearbox by Cepstral analysis, Ph.D. thesis, Jean Monnet University of St Etienne (FR), p. 141 (1999) (in French)

[21] Pareya A., El Badaoui M., Guillet F., Tandon N.. Dynamic modelling of spur gear pair and application of empirical mode decomposition-based statistical analysis for early detection of localized tooth defect, Journal of Sound and Vibration 294, 547-561 (2006) 